\title{
Stability switches in a ring-structured predator-prey metapopulation model with dispersal delay
}

Guowei Sun ${ }^{1}$ and Ali Mai ${ }^{1 *}$

"Correspondence: maialiy@126.com

'School of Mathematics and Information Technology, Yuncheng

University, Yuncheng, China

\section{Springer}

\begin{abstract}
In this paper, we consider a predator-prey metapopulation model with a ring-structured configuration of an arbitrary and finite number of patches. The prey are assumed to disperse between the connected patches with a constant dispersal delay. We show that the dispersal delay can induce stability switches exhibiting both stabilizing and destabilizing roles in the stability of the symmetric coexistence equilibrium. Numerical simulations are presented to further illustrate the effects of the dispersal delay, the dispersal rate, the fraction of dispersal due to predation avoidance and the network topology on the number of stability switches.
\end{abstract}

\section{Introduction}

The predator-prey relationship is one of the fundamental relationships in ecological systems and has been extensively studied in the literature [1]. Recently, there has been a growing interest in integrating the spatial heterogeneity into modeling metapopulation dynamics. A metacommunity is a set of local communities linked by dispersal of species. It has been shown that metacommunity dynamics can be greatly influenced by dispersal between discrete patches [2,3].

Many factors such as lack of food, competition, sex, age, climate, seasons and overpopulation in a patch contribute to the dispersal of either prey, or predator or both between patches [4]. For instance, prey may choose to move to other patches on the basis of resource availability and predation risk, while predator tend to move to patches with more prey. Many mathematical models have been proposed to study the impacts of dispersal over patches on metacommunity dynamics. For example, the dispersal of a single species is considered in [5-9], while the case with the dispersal of both prey and predator is studied in [10-15]. It has been shown that the dispersal plays an important role on the persistence and stability of the resulting ecosystems.

Since it always takes time for species to complete the dispersal from one patch to another, it is natural to incorporate the travel time into modeling. Along this line, dispersal delay is considered in [16-19] and it is shown that the dispersal delay may induce very complex dynamics. For instance, under the framework of a two-patch predator-prey model with

(c) The Author(s) 2020. This article is licensed under a Creative Commons Attribution 4.0 International License, which permits use, sharing, adaptation, distribution and reproduction in any medium or format, as long as you give appropriate credit to the original author(s) and the source, provide a link to the Creative Commons licence, and indicate if changes were made. The images or other third party material in this article are included in the article's Creative Commons licence, unless indicated otherwise in a credit line to the material. If material is not included in the article's Creative Commons licence and your intended use is not permitted by statutory regulation or exceeds the permitted use, you will need to obtain permission directly from the copyright holder. To view a copy of this licence, visit http://creativecommons.org/licenses/by/4.0/. 
delayed dispersal of predator, it is found that if the dispersal rates are in an intermediate range and the mean time that the predator spent in one patch is much shorter than the timescale of reproduction of the prey and is larger than the double mean time of capture of prey, the dispersal delay can induce stability switches such that an otherwise unstable coexistence equilibrium can be stabilized over a finite number of stability intervals [19].

Most of the aforementioned work considered the case that dispersal occurs only between two patches. If the environment involves more than two patches, then there could be many different configurations of network topology. How the impacts of dispersal differ under different configurations of network topology has not been well addressed in the literature [20]. Recently, Mai et al. considered a predator-prey metapopulation model over an arbitrary and finite number of identical patches [18], where the dispersal is assumed to occur among all patches in the environment with the same rates. Such figuration is referred to as the fully connected configuration. In this paper, we shall consider the ringstructured configuration: the patches under consideration form a ring structure, and dispersal occurs only between two neighbouring patches. We allow the number of patches to be arbitrary and finite. In order to have a comparison with the results with the fully connected configuration, we also assume that all patches are identical and the dispersal rates of the prey are the same.

The rest of the paper is organized as follows. In Sect. 2 we present the metapopulation model. The stability analysis of the symmetric coexistence equilibrium is given in Sect. 3 . A specific example is presented in Sect. 4. Some numerical simulations based on our theoretical analysis are reported in Sect. 5. We summarize our results in Sect. 6.

\section{The model}

We use a general predator-prey model given in [21] to describe the interaction between prey and predator in an isolated patch,

$$
\left\{\begin{array}{l}
\frac{d x}{d t}=x g(x)-\varepsilon y p(x) \\
\frac{d y}{d t}=y[p(x)-q(x)]
\end{array}\right.
$$

Here $x$ represents the density of prey, and $y$ denotes the density of predator. In the absence of predator, prey have the per capita growth rate $g(x) \cdot p(x)$ is the functional response, and $\varepsilon$ is the conversion ratio of loss of prey due to predation. $q(x)$ denotes the death rate (which may depend on the prey density) of predator. We assume $g(x), p(x)$ and $q(x)$ satisfy the following assumptions:

(A1) $g(0)>0$ and there exists $K>0$ such that $g(K)=0$ and $(x-K) g(x)<0$ for $x \neq K$;

(A2) $p(0)=0$ and $p^{\prime}(x)>0$ for $x>0$ with $p_{\infty}:=\lim _{x \rightarrow \infty} p(x) \leq \infty$;

(A3) $q(0)>0, q^{\prime}(x) \leq 0$ for $x \geq 0$ and $\lim _{x \rightarrow \infty} q(x)=q_{\infty}>0$ exists satisfying $q_{\infty}<p_{\infty}$. Typical functions satisfying the above assumptions include

$$
g(x)=r\left(1-\frac{x}{K}\right), \quad p(x)=\frac{a x}{1+a h x}, \quad q(x)=\frac{e x+f}{r x+s} \quad(\text { with } f r>e s),
$$

which are widely used in the literature [21, 22].

It then follows from the assumptions (A2) and (A3) that there exists a unique positive number, which we denote by $x^{*}$, such that $p\left(x^{*}\right)=q\left(x^{*}\right)$. If further, $x^{*}<K$, then (A1) implies 
that $y^{*}=x^{*} g\left(x^{*}\right) / \varepsilon p\left(x^{*}\right)>0$ and $\left(x^{*}, y^{*}\right)$ is the unique coexistence equilibrium of system (2.1).

For simplicity, we assume that all patches under the ring-structured configuration are identical and the dynamics in each patch is governed by (2.1). In addition, only prey are assumed to disperse from its habitat patch to its two neighboring patches with a dispersal rate $d$, while the predator does not move between patches. As in [17], we assume that the dispersal of prey is due to two factors: a random effect and predation avoidance, and we use $\alpha \in[0,1]$ to denote the fraction of prey dispersal due to predation avoidance. Let $\tau$ denote the finite travel time during the process of dispersal. Then we can use the following metapopulation model to describe the interactions of predator and prey under a ringstructured configuration:

$$
\left\{\begin{aligned}
\frac{d x_{i}(t)}{d t}= & x_{i}(t) g\left(x_{i}(t)\right)-\varepsilon y_{i}(t) p\left(x_{i}(t)\right)-2 d\left[\alpha \gamma y_{i}(t) p\left(x_{i}(t)\right)+(1-\alpha) x_{i}(t)\right] \\
& +d\left[\alpha \gamma y_{i+1}(t-\tau) p\left(x_{i+1}(t-\tau)\right)+(1-\alpha) x_{i+1}(t-\tau)\right] \\
& +d\left[\alpha \gamma y_{i-1}(t-\tau) p\left(x_{i-1}(t-\tau)\right)+(1-\alpha) x_{i-1}(t-\tau)\right] \\
\frac{d y_{i}(t)}{d t}= & y_{i}(t)\left[p\left(x_{i}(t)\right)-q\left(x_{i}(t)\right)\right]
\end{aligned}\right.
$$

for $i=1,2, \ldots, n, n \geq 3$, where $\gamma$ is a scaling factor proportional to $\varepsilon$. To close the ring, we define $x_{0}=x_{n}, x_{n+1}=x_{1}, y_{0}=y_{n}$ and $y_{n+1}=y_{1}$.

\section{Stability of the symmetric coexistence equilibrium}

Clearly, under our assumptions, system (2.2) always admits a symmetric coexistence equilibrium $E_{n}^{*}=\left(x^{*}, y^{*}, \ldots, x^{*}, y^{*}\right) \in R^{2 n}$ provided that $x^{*} \in(0, K)$. In this section, we are concerned with the linear stability analysis of the coexistence equilibrium $E_{n}^{*}$.

\subsection{Instantaneous dispersal: $\boldsymbol{\tau}=\mathbf{0}$}

We first consider the special case where the dispersal of prey is instantaneous, i.e., $\tau=0$. Linearizing system (2.2) at the equilibrium $E_{n}^{*}$, we obtain the associated characteristic equation $\operatorname{det} J_{n}=0$, where the $2 n \times 2 n$ matrix $J_{n}$ is given by

$$
J_{n}=\left[\begin{array}{ccccccc}
J_{1} & J_{2} & 0 & \ldots & 0 & 0 & J_{2} \\
J_{2} & J_{1} & J_{2} & \ldots & 0 & 0 & 0 \\
\vdots & \vdots & \vdots & \vdots & \vdots & \vdots & \vdots \\
0 & 0 & 0 & \ldots & J_{2} & J_{1} & J_{2} \\
J_{2} & 0 & 0 & \ldots & 0 & J_{2} & J_{1}
\end{array}\right]
$$

with

$$
J_{1}=\left[\begin{array}{cc}
\lambda-A+2 d(\alpha \gamma D+1-\alpha) & \varepsilon \mu+2 d \alpha \gamma \mu \\
-B & \lambda
\end{array}\right]
$$

and

$$
J_{2}=\left[\begin{array}{cc}
-d(\alpha \gamma D+1-\alpha) & -d \alpha \gamma \mu \\
0 & 0
\end{array}\right],
$$

where $A=g\left(x^{*}\right)+x^{*} g^{\prime}\left(x^{*}\right)-\varepsilon y^{*} p^{\prime}\left(x^{*}\right), B=y^{*}\left(p^{\prime}\left(x^{*}\right)-q^{\prime}\left(x^{*}\right)\right), D=y^{*} p^{\prime}\left(x^{*}\right), \mu=p\left(x^{*}\right)=q\left(x^{*}\right)$. It follows from (A2)-(A3) that $B>0, D>0$ and $\mu>0$. Therefore, $J_{n}$ is a block-circulant 
matrix with $2 \times 2$ blocks. Using the formula in [23], we find that

$$
\operatorname{det} J_{n}=\prod_{k=0}^{n-1} \operatorname{det}\left(J_{1}+2 J_{2} \cdot \cos \frac{2 k \pi}{n}\right) .
$$

Thus, the eigenvalues are determined by the equations

$$
\operatorname{det}\left(J_{1}+2 J_{2} \cdot \cos \frac{2 k \pi}{n}\right), \quad k=0,1, \ldots, n-1
$$

Note that $\cos (\theta)=\cos (2 \pi-\theta)$. Let [.] denote the greatest integer function. Then (3.1) contains $\left[\frac{n}{2}\right]+1$ distinct equations. For example, if $n=7$, then there are $\left[\frac{7}{2}\right]+1=4$ distinct characteristic equations, while if $n=8$, then there are $\left[\frac{8}{2}\right]+1=5$ characteristic equations. Set

$$
\mathcal{K}:=\left\{0,1,2, \ldots,\left[\frac{n}{2}\right]\right\} .
$$

In the sequel, we always assume that $k \in \mathcal{K}$. When $\tau=0$, the characteristic equations reduce to

$$
\lambda^{2}-\lambda\left(A-2 d \tilde{b}\left(1-\cos \frac{2 k \pi}{n}\right)\right)+\varepsilon \mu B+2 d \tilde{a}\left(1-\cos \frac{2 k \pi}{n}\right)=0,
$$

where $\tilde{a}=\alpha \gamma \mu B, \tilde{b}=\alpha \gamma D+1-\alpha$.

By the Routh-Hurwitz criterion [24], it is easy to obtain the following result.

Lemma 3.1 Consider system (2.2) with $\tau=0$.

(1) If $A<0$, the coexistence equilibrium $E_{n}^{*}$ is locally asymptotically stable.

(2) If $A>0$, the coexistence equilibrium $E_{n}^{*}$ is unstable.

Proof If $A<0$, then $A-2 d \tilde{b}\left(1-\cos \frac{2 k \pi}{n}\right)<0$. Therefore, all characteristic roots of (3.3) have negative real parts and hence $E_{n}^{*}$ is locally asymptotically stable. If $A>0$, then (3.3) with $k=0$ admits two roots with positive real parts. Thus $E_{n}^{*}$ is unstable.

Remark 3.2 Note that the stability condition of the coexistence equilibrium of the ringstructured system is the same as that of the coexistence equilibrium of the single-patch system. This indicates that the instantaneous dispersal of prey does not affect the stability of the coexistence equilibrium.

\subsection{Random dispersal with delay: $\tau>0$ and $\alpha=0$}

Linearizing system (2.2) at the equilibrium $E_{n}^{*}$, we obtain the characteristic equations of system (2.2):

$$
\lambda^{2}-\lambda(A-2 d \tilde{b})-2 \lambda d \tilde{b} \cos \frac{2 k \pi}{n} e^{-\lambda \tau}+\varepsilon \mu B+2 d \tilde{a}-2 d \tilde{a} \cos \frac{2 k \pi}{n} e^{-\lambda \tau}=0,
$$

where $k \in \mathcal{K}$. When $\alpha=0$, the characteristic equations (3.4) become

$$
\lambda^{2}-\lambda(A-2 d)-2 \lambda d \cos \frac{2 k \pi}{n} e^{-\lambda \tau}+\varepsilon \mu B=0
$$


It is well known that the coexistence equilibrium $E_{n}^{*}$ of system (2.2) is stable if and only if all characteristic roots of (3.5) have negative real parts [25]. To explore how the dispersal delay affects the dynamics of the coexistence equilibrium, next we use the dispersal delay $\tau$ as the bifurcation parameter. As $\tau$ increases, the stability of the equilibrium changes only when some characteristic roots cross the imaginary axis transversely [25-27]. It is clear that zero is not a characteristic root of (3.5), we thus look for purely imaginary roots. Since complex roots appear in pairs, we assume that $\lambda=i \omega$, with $\omega>0$. Substituting $\lambda=i \omega$ into (3.5), and separating the real and imaginary parts, we obtain

$$
\left\{\begin{array}{l}
2 d-A=2 d \cos \omega \tau \cdot \cos \frac{2 k \pi}{n}, \\
-\omega^{2}+\varepsilon \mu B=2 \omega d \sin \omega \tau \cdot \cos \frac{2 k \pi}{n} .
\end{array}\right.
$$

From (3.6), we have

$$
\frac{\left(\varepsilon \mu B-\omega^{2}\right)^{2}}{\omega^{2}}=\left(2 d \cos \frac{2 k \pi}{n}\right)^{2}-(2 d-A)^{2}:=C_{k} .
$$

If $C_{k}>0$, then (3.7) admits the following two positive roots:

$$
\omega_{1 k}=\frac{-\sqrt{C_{k}}+\sqrt{C_{k}+4 \varepsilon \mu B}}{2}, \quad \omega_{2 k}=\frac{\sqrt{C_{k}}+\sqrt{C_{k}+4 \varepsilon \mu B}}{2} .
$$

Lemma 3.3 Suppose at certain $\tau$, the characteristic equations (3.5) have a pair of purely imaginary roots $\pm i \omega_{j k}(j=1$ or $j=2$ and $k \in \mathcal{K})$. Then

$$
\left.\frac{d \operatorname{Re}(\lambda)}{d \tau}\right|_{\lambda=i \omega_{1 k}}<0,\left.\quad \frac{d \operatorname{Re}(\lambda)}{d \tau}\right|_{\lambda=i \omega_{2 k}}>0
$$

Proof It follows from (3.5) that

$$
\frac{d \lambda}{d \tau}=-\frac{2 \lambda^{2} d \cos \frac{2 k \pi}{n} e^{-\lambda \tau}}{2 \lambda-(A-2 d)-2 d \cos \frac{2 k \pi}{n} e^{-\lambda \tau}+2 \lambda \tau \cos \frac{2 k \pi}{n} d e^{-\lambda \tau}} .
$$

By (3.6), we further have

$$
\left.\operatorname{sign}\left(\frac{d \operatorname{Re}(\lambda)}{d \tau}\right)\right|_{\lambda=i \omega}=\operatorname{sign}\left(-2\left(\varepsilon \mu B-\omega^{2}\right)-C_{k}\right) .
$$

Note that $\varepsilon \mu B-\omega_{1 k}^{2}=\omega_{1 k} \sqrt{C_{k}}$ and $\varepsilon \mu B-\omega_{2 k}^{2}=-\omega_{2 k} \sqrt{C_{k}}$, then

$$
\left.\operatorname{sign}\left(\frac{d \operatorname{Re}(\lambda)}{d \tau}\right)\right|_{\lambda=i \omega_{1 k}}=\operatorname{sign}\left(-2 \omega_{1 k} \sqrt{C_{k}}-C_{k}\right)
$$

and

$$
\begin{aligned}
\left.\operatorname{sign}\left(\frac{d \operatorname{Re}(\lambda)}{d \tau}\right)\right|_{\lambda=i \omega_{2 k}} & =\operatorname{sign}\left(\left(\sqrt{C_{k}}+\sqrt{C_{k}+4 \varepsilon \mu B}\right) \sqrt{C_{k}}-C_{k}\right) \\
& =\operatorname{sign}\left(\sqrt{C_{k}^{2}+4 \varepsilon \mu B C_{k}}\right)
\end{aligned}
$$

Then the conclusion follows immediately. 
To analyze the stability of the system (2.2) at $E_{n}^{*}$, next we consider two cases: $A<0$ and $A>0$, respectively.

Case 1: $A<0$.

$A<0$ implies that $C_{k}<0$ for all $k \in \mathcal{K}$. Thus (3.7) does not admit any solutions. That is, no characteristic roots of (3.4) would cross the imaginary axis. By Lemma 3.1, $E_{n}^{*}$ is stable with $\tau=0$, thus, $E_{n}^{*}$ remains stable for $\tau>0$.

Case 2: $A>0$.

In this case, the coexistence equilibrium $E_{n}^{*}$ is unstable when $\tau=0$ by Lemma 3.1. Next we examine if the unstable coexistence equilibrium $E_{n}^{*}$ still remains unstable as $\tau$ increases, or can it be stabilized for some values of $\tau$. To this end, we consider three cases: (i) $0<d<\frac{A}{4}$; (ii) $\frac{A}{4}<d<\frac{A}{2}$; (iii) $d>\frac{A}{2}$.

We first consider (i) $0<d<\frac{A}{4}$.

In this case, it follows from (3.7) that $C_{k}<0$ for all $k \in \mathcal{K}$. Therefore, there are no such purely imaginary roots. Consequently, the coexistence equilibrium $E_{n}^{*}$ remains unstable as $\tau$ increases.

(ii) $\frac{A}{4}<d<\frac{A}{2}$.

In this case, there exists some $k \in \mathcal{K}$ such that $C_{k}>0$ provided that $\left|\cos \frac{2 k \pi}{n}\right|>\left|\frac{A-2 d}{2 d}\right|$. For such $k$, (3.7) admits two positive solutions: $\omega_{1 k}$ and $\omega_{2 k}$, given in (3.8). Therefore, we distinguish three different conditions: (1) $\cos \frac{2 k \pi}{n}>\frac{A-2 d}{2 d}>0$; (2) $\cos \frac{2 k \pi}{n}<\frac{2 d-A}{2 d}<0$; (3) $\frac{2 d-A}{2 d}<\cos \frac{2 k \pi}{n}<\frac{A-2 d}{2 d}$;

We denote by $n(\mathcal{M})$ the number of elements in the set $\mathcal{M}$. Let

$$
\begin{aligned}
& \mathcal{K}_{1}=\left\{k \in \mathcal{K} \mid \cos \frac{2 k \pi}{n}>\frac{A-2 d}{2 d}>0\right\}, \\
& \mathcal{K}_{2}=\left\{k \in \mathcal{K} \mid \cos \frac{2 k \pi}{n}<\frac{2 d-A}{2 d}<0\right\}, \\
& \mathcal{K}_{3}=\left\{k \in \mathcal{K} \mid \frac{2 d-A}{2 d}<\cos \frac{2 k \pi}{n}<\frac{A-2 d}{2 d}\right\} .
\end{aligned}
$$

Under condition (1), there are $n\left(\mathcal{K}_{1}\right)$ characteristic equations (3.5). For these characteristic equations, together with (3.6), we have $-1<\cos \omega \tau<0$. Then

$$
\omega \tau \in\left(\frac{\pi}{2}+2 l \pi, \frac{3 \pi}{2}+2 l \pi\right), \quad l=0,1, \ldots
$$

Note that

$$
\sin \omega_{1 k} \tau=\frac{\sqrt{C_{k}}}{2 d} \cdot \frac{1}{\cos \frac{2 k \pi}{n}} \quad \text { and } \quad \sin \omega_{2 k} \tau=-\frac{\sqrt{C_{k}}}{2 d} \cdot \frac{1}{\cos \frac{2 k \pi}{n}} .
$$

Thus, $\sin \omega_{1 k} \tau>0$ and $\sin \omega_{2 k} \tau<0$.

We define

$$
\theta_{1}^{(k)}=\cos ^{-1}\left(\frac{2 d-A}{2 d \cos \frac{2 k \pi}{n}}\right) \quad \text { and } \quad \theta_{2}^{(k)}=2 \pi-\theta_{1}^{(k)}, \quad k \in \mathcal{K}_{1} .
$$

Then $\theta_{1}^{(k)} \in\left(\frac{\pi}{2}, \pi\right)$ and $\theta_{2}^{(k)} \in\left(\pi, \frac{3 \pi}{2}\right)$. This shows that purely imaginary roots of the characteristic equations (3.5) under condition (1) are obtained at the following sequences of 
positive values of $\tau$ :

$$
\tau_{l, 1}^{(k)}=\frac{\theta_{1}^{(k)}+2 l \pi}{\omega_{1 k}}, \quad \tau_{l, 2}^{(k)}=\frac{\theta_{2}^{(k)}+2 l \pi}{\omega_{2 k}},
$$

where $k \in \mathcal{K}_{1}, l=0,1,2, \ldots$

It follows from $\left.\frac{d \operatorname{Re}(\lambda)}{d \tau}\right|_{\lambda=i \omega_{1 k}}<0$ and $\left.\frac{d \operatorname{Re}(\lambda)}{d \tau}\right|_{\lambda=i \omega_{2 k}}>0$ (Lemma 3.3) that, as $\tau$ increases, the characteristic roots cross the imaginary axis through $\pm i \omega_{1 k}$ at $\tau=\tau_{l, 1}^{(k)}$ from right to left and the number of characteristic roots with positive real parts is reduced by 2 . The characteristic roots cross the imaginary axis through $\pm i \omega_{2 k}$ at $\tau=\tau_{l, 2}^{(k)}$ from left to right and the number of characteristic roots with positive real parts is increased by 2 .

Similarly, under condition (2), there are $n\left(\mathcal{K}_{2}\right)$ characteristic equations (3.5) satisfying $\cos \frac{2 k \pi}{n}<\frac{2 d-A}{2 d}<0$. For these characteristic equations, in a similar manner, we can show that there are purely imaginary roots at the following sequences of (Hopf bifurcation) values of $\tau$ :

$$
\tau_{l, 3}^{(k)}=\frac{\theta_{3}^{(k)}+2 l \pi}{\omega_{1 k}}, \quad \tau_{l, 4}^{(k)}=\frac{\theta_{4}^{(k)}+2 l \pi}{\omega_{2 k}},
$$

with

$$
\theta_{3}^{(k)}=2 \pi-\cos ^{-1}\left(\frac{2 d-A}{2 d \cos \frac{2 k \pi}{n}}\right) \in\left(\frac{3 \pi}{2}, 2 \pi\right), \quad \theta_{4}^{(k)}=\cos ^{-1}\left(\frac{2 d-A}{2 d \cos \frac{2 k \pi}{n}}\right) \in\left(0, \frac{\pi}{2}\right),
$$

where $k \in \mathcal{K}_{2}, l=0,1,2, \ldots$.

By Lemma 3.3, as $\tau$ increases, the characteristic roots cross the imaginary axis through $\pm i \omega_{1 k}$ at $\tau=\tau_{l, 3}^{(k)}$ from right to left and the number of characteristic roots with positive real parts is decreased by 2 , while the crossing through $\pm i \omega_{2 k}$ occurs at $\tau=\tau_{l, 4}^{(k)}$ from left to right, and the number of characteristic roots with positive real parts is increased by 2 .

Finally, condition (3) leads to $\frac{2 d-A}{2 d}<\cos \frac{2 k \pi}{n}<\frac{A-2 d}{2 d}$ and $C_{k}<0$ for $k \in \mathcal{K}_{3}$, then these $n\left(\mathcal{K}_{3}\right)$ characteristic equations have no purely imaginary roots.

(iii) $d>\frac{A}{2}$

In this case, we also have three cases to consider: (4) $\cos \frac{2 k \pi}{n}>\frac{2 d-A}{2 d}>0$; (5) $\cos \frac{2 k \pi}{n}<$ $\frac{A-2 d}{2 d}<0$; (6) $\frac{A-2 d}{2 d}<\cos \frac{2 k \pi}{n}<\frac{2 d-A}{2 d}$.

We define

$$
\begin{aligned}
& \mathcal{K}_{4}=\left\{k \in \mathcal{K} \mid \cos \frac{2 k \pi}{n}>\frac{2 d-A}{2 d}>0\right\}, \\
& \mathcal{K}_{5}=\left\{k \in \mathcal{K} \mid \cos \frac{2 k \pi}{n}<\frac{A-2 d}{2 d}<0\right\}, \\
& \mathcal{K}_{6}=\left\{k \in \mathcal{K} \mid \frac{A-2 d}{2 d}<\cos \frac{2 k \pi}{n}<\frac{2 d-A}{2 d}\right\} .
\end{aligned}
$$

Using a similar procedure to case (ii), for condition (4), we obtain sequences $\left\{\tau_{l, 5}^{(k)}\right\}$ and $\left\{\tau_{l, 6}^{(k)}\right\}$ defined by

$$
\tau_{l, 5}^{(k)}=\frac{\theta_{5}^{(k)}+2 l \pi}{\omega_{1 k}}, \quad \tau_{l, 6}^{(k)}=\frac{\theta_{6}^{(k)}+2 l \pi}{\omega_{2 k}},
$$


with

$$
\theta_{5}^{(k)}=\cos ^{-1}\left(\frac{2 d-A}{2 d \cos \frac{2 k \pi}{n}}\right) \in(0, \pi / 2), \quad \theta_{6}^{(k)}=2 \pi-\theta_{5} \in(3 \pi / 2,2 \pi),
$$

where $\omega_{1 k}$ and $\omega_{2 k}$ are defined in (3.7) and $k \in \mathcal{K}_{4}, l=0,1,2, \ldots$. Similarly, under condition (5), we obtain the sequences $\left\{\tau_{l, 7}^{(k)}\right\}$ and $\left\{\tau_{l, 8}^{(k)}\right\}$ defined by

$$
\tau_{l, 7}^{(k)}=\frac{\theta_{7}^{(k)}+2 l \pi}{\omega_{1 k}}, \quad \tau_{l, 8}^{(k)}=\frac{\theta_{8}^{(k)}+2 l \pi}{\omega_{2 k}},
$$

with

$$
\theta_{7}^{(k)}=2 \pi-\cos ^{-1}\left(\frac{2 d-A}{2 d \cos \frac{2 k \pi}{n}}\right) \in(\pi, 3 \pi / 2), \quad \theta_{8}^{(k)}=\cos ^{-1}\left(\frac{2 d-A}{2 d \cos \frac{2 k \pi}{n}}\right) \in(\pi / 2, \pi),
$$

where $\omega_{1 k}$ and $\omega_{2 k}$ are defined in (3.7) and $k \in \mathcal{K}_{5}, l=0,1,2, \ldots$. Under condition (6), it is easy to show that characteristic equations (3.5) with $k \in \mathcal{K}_{6}$ have no purely imaginary roots.

From the above analysis, we see that, for case (ii), $n\left(\mathcal{K}_{3}\right)$ characteristic equations have no purely imaginary roots; while for $n\left(\mathcal{K}_{1}\right)+n\left(\mathcal{K}_{2}\right)$ characteristic equations, there are sequences of critical values of $\tau$ at which the characteristic roots cross the imaginary axis (either from left to right or from right to left). For case (iii), $n\left(\mathcal{K}_{6}\right)$ characteristic equations have no purely imaginary roots; while for $n\left(\mathcal{K}_{4}\right)+n\left(\mathcal{K}_{5}\right)$ characteristic equations, there are sequences of critical values of $\tau$ at which the characteristic roots cross the imaginary axis.

To further investigate the possibility of undergoing stability switches, we introduce some notation. For the above obtained sequences of critical values of $\tau$, we define

$$
\Gamma_{j}=\left\{\tau_{0, j}^{(k)}, \tau_{1, j}^{(k)}, \ldots, \tau_{k, j}^{(k)}, \ldots\right\}, \quad j=1,2,3,4,5,6,7,8
$$

where $k \in \mathcal{K}_{1}$ if $j=1,2 ; k \in \mathcal{K}_{2}$ if $j=3,4 ; k \in \mathcal{K}_{4}$ if $j=5,6$ and $k \in \mathcal{K}_{5}$ if $j=7,8$.

For each case (case (ii) and case (iii)), we sort the obtained sequences of $\tau$ as a single increasing sequence

$$
\left\{\tau_{j}\right\}_{j=1}^{\infty}=\left\{\tau_{1}, \tau_{2}, \tau_{3}, \ldots, \tau_{j}, \ldots\right\}
$$

with $\tau_{j}<\tau_{j+1}, j=1,2, \ldots$ For instance, if $n\left(\mathcal{K}_{1}\right)=1, n\left(\mathcal{K}_{2}\right)=1$ and $\tau_{0,4}^{(2)}<\tau_{0,1}^{(0)}<\tau_{0,2}^{(0)}<\tau_{0,3}^{(2)}<$ $\cdots$, then the new sequence reads

$$
\left\{\tau_{j}\right\}_{j=1}^{\infty}=\left\{\tau_{1}=\tau_{0,4}^{(2)}, \tau_{2}=\tau_{0,1}^{(0)}, \tau_{3}=\tau_{0,2}^{(0)}, \tau_{4}=\tau_{0,3}^{(2)}, \ldots\right\} .
$$

We associate each element $\tau_{j}$ of the sequence with a number $\sigma_{j}$ defined by

$$
\sigma_{j}=\left\{\begin{array}{ll}
+1, & \text { if } \tau_{j} \in \Gamma_{2} \cup \Gamma_{4} \cup \Gamma_{6} \cup \Gamma_{8}, \\
-1, & \text { if } \tau_{j} \in \Gamma_{1} \cup \Gamma_{3} \cup \Gamma_{5} \cup \Gamma_{7},
\end{array} \quad j=1,2, \ldots\right.
$$

At $\tau=\tau_{j}$, if $\sigma_{j}=+1$, then it means a pair of purely imaginary roots cross the imaginary axis at $\tau=\tau_{j}$ from left to right, and the number of characteristic roots with positive real 
parts increases by 2 and we say the delay $\tau_{j}$ has a positive jump, otherwise, if $\sigma_{j}=-1$, then a pair of purely imaginary roots cross the imaginary axis at $\tau=\tau_{j}$ from right to left, and the number of characteristic roots with positive real parts is reduced by 2 and we say the delay $\tau_{j}$ has a negative jump.

Define a sequence $\left\{s_{j}\right\}_{j=1}^{\infty}$ as

$$
s_{j}=\sum_{k=1}^{j} \sigma_{k}
$$

Note that characteristic equations (3.5) with $\tau=0$ reduce to

$$
\lambda^{2}-\lambda\left(A-2 d\left(1-\cos \frac{2 k \pi}{n}\right)\right)+\varepsilon \mu B=0, \quad k \in \mathcal{K} .
$$

Here, we denote by $n(\operatorname{Re}(\lambda)>0)$ the number of characteristic roots with positive real parts of characteristic equation (3.9).

First, we consider the three cases of $k$ for case (ii). If $k \in \mathcal{K}_{1}$, then $\cos \frac{2 k \pi}{n}>\frac{A-2 d}{2 d}$ and $A-2 d\left(1-\cos \frac{2 k \pi}{n}\right)>0$. So $n(\operatorname{Re}(\lambda)>0)=2 n\left(\mathcal{K}_{1}\right)$; If $k \in \mathcal{K}_{2}$, then $\cos \frac{2 k \pi}{n}<\frac{2 d-A}{2 d}$ and $A-$ $2 d\left(1-\cos \frac{2 k \pi}{n}\right)<0$. Thus, $n(\operatorname{Re}(\lambda)>0)=0$; If $k \in \mathcal{K}_{3}$, then $\frac{2 d-A}{2 d}<\cos \frac{2 k \pi}{n}<\frac{A-2 d}{2 d}$ and $A-$ $2 d\left(1-\cos \frac{2 k \pi}{n}\right)>0$. Therefore, $n(\operatorname{Re}(\lambda)>0)=2 n\left(\mathcal{K}_{3}\right)$.

We define the set $S_{2}$ as

$$
S_{2}=\left\{s_{j} \mid s_{j}=-n\left(\mathcal{K}_{1}\right)-n\left(\mathcal{K}_{3}\right), j \in\{1,2, \ldots\}\right\} .
$$

Note that, for case (ii), when $\tau=0$, the coexistence equilibrium $E_{n}^{*}$ is unstable and there are $2\left(n\left(\mathcal{K}_{1}\right)+n\left(\mathcal{K}_{3}\right)\right)$ characteristic roots with positive real parts. Thus there are $2\left(n\left(\mathcal{K}_{1}\right)+\right.$ $\left.n\left(\mathcal{K}_{3}\right)+s_{j}\right)$ characteristic roots with positive real parts for $\tau \in\left(\tau_{j}, \tau_{j+1}\right), j=1,2, \ldots$ Therefore, if for some $j \in\{1,2, \ldots\}, s_{j}=-n\left(\mathcal{K}_{1}\right)-n\left(\mathcal{K}_{3}\right)$, then the coexistence equilibrium $E_{n}^{*}$ is locally asymptotically stable for $\tau$ in the interval $\left(\tau_{j}, \tau_{j+1}\right)$, and if $s_{j} \geq-n\left(\mathcal{K}_{1}\right)-n\left(\mathcal{K}_{3}\right)+1$, then the coexistence equilibrium $E_{n}^{*}$ is unstable for $\tau \in\left(\tau_{j}, \tau_{j+1}\right)$.

Similarly, we define the set $S_{3}$ as

$$
S_{3}=\left\{s_{j} \mid s_{j}=-n\left(\mathcal{K}_{4}\right), j \in\{1,2, \ldots\}\right\} .
$$

Note that, for case (iii), the number of characteristic roots with positive real parts for characteristic equations (3.9) under the conditions (4)-(6) is $n(\operatorname{Re}(\lambda)>0)=2 n\left(\mathcal{K}_{4}\right)$ by using a similar calculating method to case (ii). Thus there are $2\left(n\left(\mathcal{K}_{4}\right)+s_{j}\right)$ characteristic roots with positive real parts for $\tau \in\left(\tau_{j}, \tau_{j+1}\right), j=1,2, \ldots$. Consequently, if for some $j \in\{1,2, \ldots\}$, $s_{j}=-n\left(\mathcal{K}_{4}\right)$, then the coexistence equilibrium $E_{n}^{*}$ is locally asymptotically stable for $\tau$ in the interval $\left(\tau_{j}, \tau_{j+1}\right)$, and if $s_{j} \geq-n\left(\mathcal{K}_{4}\right)+1$, then the coexistence equilibrium $E_{n}^{*}$ is unstable for $\tau \in\left(\tau_{j}, \tau_{j+1}\right)$.

We denote by $\mathcal{N}$ the number of elements in the set $S_{j}, j=2$ or $j=3$, i.e.,

$$
\mathcal{N}=n\left(S_{j}\right), \quad j=2,3
$$

Then $\mathcal{N}$ determines the number of stability intervals. 


\subsection{Predator-induced dispersal with delay: $\tau>0$ and $\alpha>0$}

In this section, we study the stability of the coexistence equilibrium $E_{n}^{*}$ of system (2.2) with $\alpha>0$ and $\tau>0$.

For any fixed $\alpha>0$, we use the same method as for the case with $\alpha=0$ to find sequences of critical values of $\tau$ at which the characteristic equations (3.4) admit purely imaginary characteristic roots, and to compute directions of the characteristic roots crossing the imaginary axis and then to determine if there are stability intervals.

Following the results in Sect. 5 of Cooke and Grossman [26], the characteristic equation (3.4) admits purely imaginary roots if and only if the following inequalities hold:

$$
2 \varepsilon \mu B+\tilde{C}_{k}>2 \sqrt{(\varepsilon \mu B)^{2}+4 d \varepsilon \mu B \tilde{a}+(2 d \tilde{a})^{2} \sin ^{2} \frac{2 k \pi}{n}},
$$

where $k \in \mathcal{K}, \tilde{C}_{k}=4(\tilde{a}+A \tilde{b}) d-A^{2}-(2 d \tilde{b})^{2} \sin ^{2} \frac{2 k \pi}{n}, \tilde{a}=\alpha \gamma \mu B>0$ and $\tilde{b}=\alpha \gamma D+1-\alpha>0$. Clearly if $0<d<\frac{A^{2}}{4(\tilde{a}+A \tilde{b})}$, then $\tilde{C}_{k} \leq 0$, for all $k \in \mathcal{K}$, (3.13) can never hold, thus, all characteristic equations (3.4) have no purely imaginary roots. If $d>\frac{A^{2}}{4(\tilde{a}+A \tilde{b})}$ and $\tilde{C}_{k}>0$, then it is possible for characteristic equations (3.4) with some $k \in \mathcal{K}$ to admit purely imaginary roots and thus the interplay of $\alpha$ and $\tau$ may affect the stability of coexistence equilibrium. Substituting $\lambda=i \tilde{\omega}$ (with $\tilde{\omega}>0$ ) into (3.4) and separating the real and imaginary parts, we obtain

$$
\left\{\begin{array}{l}
\cos \tilde{\omega} \tau=\frac{\tilde{a} \varepsilon \mu B+2 d \tilde{a}^{2}+\tilde{\omega}^{2}\left[2 d \tilde{b}^{2}-A \tilde{b}-\tilde{a}\right]}{2 d \cos \frac{2 k \pi}{n}\left(\tilde{b}^{2} \tilde{\omega}^{2}+\tilde{a}^{2}\right)}, \\
\sin \tilde{\omega} \tau=\tilde{\omega} \frac{\tilde{b}\left(\varepsilon \mu B-\tilde{\omega}^{2}\right)+\tilde{a} A}{2 d \cos \frac{2 k \pi}{n}\left(\tilde{b}^{2} \tilde{\omega}^{2}+\tilde{a}^{2}\right)} .
\end{array}\right.
$$

Squaring and adding these two equations, and solving the resulting quadratic equation of $\tilde{\omega}^{2}$, we obtain

$$
\tilde{\omega}_{1 k}=\sqrt{\frac{1}{2}\left(\tilde{C}_{k}+2 \varepsilon \mu B-\sqrt{\Delta_{k}}\right)}, \quad \tilde{\omega}_{2 k}=\sqrt{\frac{1}{2}\left(\tilde{C}_{k}+2 \varepsilon \mu B+\sqrt{\Delta_{k}}\right)}
$$

provided that $\Delta_{k}:=\tilde{C}_{k}^{2}+4 \varepsilon \mu B \tilde{C}_{k}-16 d \varepsilon \mu B \tilde{a}-16(d \tilde{a})^{2}\left(1-\cos ^{2}\left(\frac{2 k \pi}{n}\right)\right)>0$. The associated two sequences of critical values of $\tau$ are determined by

$$
\tilde{\tau}_{l, 1}^{(k)}=\frac{\tilde{\theta}_{1}^{(k)}+2 l \pi}{\tilde{\omega}_{1 k}}, \quad \tilde{\tau}_{l, 2}^{(k)}=\frac{\tilde{\theta}_{2}^{(k)}+2 l \pi}{\tilde{\omega}_{2 k}}, \quad l=0,1,2, \ldots
$$

where

$$
\tilde{\theta}_{1}^{(k)}:= \begin{cases}\tilde{\xi}_{1}^{(k)}, & \text { if } \frac{\tilde{a} A+\tilde{b}\left(\varepsilon \mu B-\tilde{\omega}_{1 k}^{2}\right)}{\cos \frac{2 k \pi}{n}}>0, \\ 2 \pi-\tilde{\xi}_{1}^{(k)}, & \text { if } \frac{\tilde{a} A+\tilde{b}\left(\varepsilon \mu B-\tilde{\omega}_{1 k}^{2}\right)}{\cos \frac{2 k \pi}{n}}<0\end{cases}
$$

and

$$
\tilde{\theta}_{2}^{(k)}:= \begin{cases}\tilde{\xi}_{2}^{(k)}, & \text { if } \frac{\tilde{a} A+\tilde{b}\left(\varepsilon \mu \beta-\tilde{\omega}_{2 k}^{2}\right)}{\cos \frac{2 k \pi}{n}}>0, \\ 2 \pi-\tilde{\xi}_{2}^{(k)}, & \text { if } \frac{\tilde{a} A+\tilde{b}\left(\varepsilon \mu B-\tilde{\omega}_{2 k}^{2}\right)}{\cos \frac{2 k \pi}{n}}<0,\end{cases}
$$


with

$$
\tilde{\xi}_{1}^{(k)}=\cos ^{-1}\left(\frac{\tilde{a} \varepsilon \mu B+2 d \tilde{a}^{2}+\tilde{\omega}_{1 k}^{2}\left[2 d \tilde{b}^{2}-A \tilde{b}-\tilde{a}\right]}{2 d \cos \frac{2 k \pi}{n}\left(\tilde{b}^{2} \tilde{\omega}_{1 k}^{2}+\tilde{a}^{2}\right)}\right)
$$

and

$$
\tilde{\xi}_{2}^{(k)}=\cos ^{-1}\left(\frac{\tilde{a} \varepsilon \mu B+2 d \tilde{a}^{2}+\tilde{\omega}_{2 k}^{2}\left[2 d \tilde{b}^{2}-A \tilde{b}-\tilde{a}\right]}{2 d \cos \frac{2 k \pi}{n}\left(\tilde{b}^{2} \tilde{\omega}_{2 k}^{2}+\tilde{a}^{2}\right)}\right) .
$$

Moreover, we can calculate

$$
\left.\operatorname{sign} \frac{d \operatorname{Re}(\lambda)}{d \tau}\right|_{\lambda=i \tilde{\omega}_{1 k}}=\operatorname{sign}\left(-\sqrt{\Delta_{k}}\right)
$$

and

$$
\left.\operatorname{sign} \frac{d \operatorname{Re}(\lambda)}{d \tau}\right|_{\lambda=i \tilde{\omega}_{2 k}}=\operatorname{sign}\left(\sqrt{\Delta_{k}}\right) .
$$

Thus, $\left.\frac{d \operatorname{Re}(\lambda)}{d \tau}\right|_{\lambda=i \tilde{\omega}_{1 k}}<0$ and $\left.\frac{d \operatorname{Re}(\lambda)}{d \tau}\right|_{\lambda=i \tilde{\omega}_{2 k}}>0$.

Similar to the case with $\alpha=0$, we define

$$
\begin{aligned}
& \tilde{\mathcal{K}}=\left\{k \in \mathcal{K} \mid \tilde{C}_{k}>0 \text { and }(3.13) \text { holds, } k \in \mathcal{K}\right\} \\
& \tilde{\Gamma}_{j}=\left\{\tilde{\tau}_{0, j}^{(k)}, \tilde{\tau}_{1, j}^{(k)}, \ldots, \tilde{\tau}_{k, j}^{(k)}, \ldots\right\}, \quad j=1,2, k \in \tilde{\mathcal{K}}
\end{aligned}
$$

and then sort the obtained sequences of $\tau$ as a single increasing sequence

$$
\left\{\tilde{\tau}_{j}\right\}_{j=1}^{\infty}=\left\{\tilde{\tau}_{1}, \tilde{\tau}_{2}, \tilde{\tau}_{3}, \ldots\right\}
$$

with $\tilde{\tau}_{j}<\tilde{\tau}_{j+1}, j=1,2, \ldots$. The corresponding $\tilde{\sigma}_{j}$ are defined as

$$
\tilde{\sigma}_{j}=\left\{\begin{array}{ll}
+1, & \text { if } \tau_{j} \in \tilde{\Gamma}_{2}, \\
-1, & \text { if } \tau_{j} \in \tilde{\Gamma}_{1},
\end{array} \quad j=1,2, \ldots\right.
$$

and the $\tilde{s}_{j}$ are defined by

$$
\tilde{s}_{j}=\sum_{k=1}^{j} \tilde{\sigma}_{k}
$$

To determine the number of stability intervals for the case with $\alpha>0$, we discuss three cases: (i) $A<0$, (ii) $A>0$ and $A-4 d \tilde{b}<0$ and (iii) $A>0$ and $A-4 d \tilde{b}>0$. Define

$$
\tilde{\mathcal{K}}_{0}=\left\{k \mid A-2 d \tilde{b}\left(1-\cos \frac{2 k \pi}{n}\right)>0\right\} .
$$

Note that when $\tau=0$, for case (i), all characteristic roots have negative real parts, for case (ii), there are $2 n\left(\tilde{\mathcal{K}}_{0}\right)$ characteristic roots with positive real parts, and for case (iii), 
there are $2\left[\frac{n}{2}\right]+2$ characteristic roots with positive real parts. Therefore, we can define the set $\tilde{S}$ as

$$
\tilde{S}= \begin{cases}\left\{s_{j} \mid s_{j}=0, j \in\{1,2, \ldots\}\right\}, & \text { if } A<0, \\ \left\{s_{j} \mid s_{j}=-n\left(\tilde{\mathcal{K}}_{0}\right), j \in\{1,2, \ldots\}\right\}, & \text { if } A>0 \text { and } A-4 d \tilde{b}<0, \\ \left\{s_{j} \mid s_{j}=-\left[\frac{n}{2}\right]-1, j \in\{1,2, \ldots\}\right\}, & \text { if } A>0 \text { and } A-4 d \tilde{b}>0 .\end{cases}
$$

Then we denote by $\tilde{\mathcal{N}}$ the number of elements in the set $\tilde{S}$, which gives the number of stability intervals.

\section{An example}

In this section, we give an example to illustrate our theoretical analysis in detail. Take $n=4$. Then the characteristic equations (3.4) of system (2.2) reduce to

$$
\lambda^{2}-\lambda(A-2 d \tilde{b})-2 \lambda d \tilde{b} \cos \frac{2 k \pi}{n} e^{-\lambda \tau}+\varepsilon \mu B+2 d \tilde{a}-2 d \tilde{a} \cos \frac{2 k \pi}{n} e^{-\lambda \tau}=0
$$

where $k=0,1,2$.

When $\alpha=0$, the characteristic equations (4.1) are given by

$$
\begin{aligned}
& \lambda^{2}-\lambda(A-2 d)-2 \lambda d e^{-\lambda \tau}+\varepsilon \mu B=0, \\
& \lambda^{2}-\lambda(A-2 d)+\varepsilon \mu B=0, \\
& \lambda^{2}-\lambda(A-2 d)+2 \lambda d e^{-\lambda \tau}+\varepsilon \mu B=0,
\end{aligned}
$$

By (3.7) and (3.8), we have $C_{0}=C_{2}=A(4 d-A)$ and $C_{1}=-(2 d-A)^{2}<0$ and then $\omega_{1}:=$ $\omega_{10}=\omega_{12}=\frac{-\sqrt{C_{0}}+\sqrt{C_{0}+4 \varepsilon \mu B}}{2}, \omega_{2}:=\omega_{20}=\omega_{22}=\frac{\sqrt{C_{0}}+\sqrt{C_{0}+4 \varepsilon \mu B}}{2}$.

As analyzed in Sect. 3 , if $A<0$, then $C_{k}<0,(k=0,2)$ and the characteristic equations (4.2) and (4.4) do not admit any purely imaginary roots. Consequently, the coexistence equilibrium $E_{4}^{*}$ remains locally asymptotically stable for any values of $\tau>0$. If $A>0$ and $0<$ $d<A / 4, C_{k}<0,(k=0,2)$, then the coexistence equilibrium $E_{4}^{*}$ remains unstable for $\tau>0$. While if $A / 4<d<A / 2$ and $d>A / 2$, we have $C_{0}=C_{2}>0$, then characteristic equations (4.2) and (4.4) can have purely imaginary roots, and thus the delay can affect the stability of the coexistence equilibrium for system (2.2) with $n=4$. Next, we discuss two cases: $A / 4<d<A / 2$ and $d>A / 2$.

If $A / 4<d<A / 2$, then the characteristic equation (4.2) admits a pair of purely imaginary roots at the following two sequences of values of $\tau$ :

$$
\tau_{l, 1}^{(0)}=\frac{\theta_{1}^{(0)}+2 l \pi}{\omega_{10}}, \tau_{l, 2}^{(0)}=\frac{\theta_{2}^{(0)}+2 l \pi}{\omega_{20}}, \quad l=0,1,2, \ldots
$$

with

$$
\theta_{1}^{(0)}=\cos ^{-1}\left(\frac{2 d-A}{2 d}\right) \in\left(\frac{\pi}{2}, \pi\right) \quad \text { and } \quad \theta_{2}^{(0)}=2 \pi-\theta_{1}^{(0)} \in\left(\pi, \frac{3 \pi}{2}\right) .
$$

For (4.4), we obtain two sequences of positive values of $\tau$ :

$$
\tau_{l, 3}^{(2)}=\frac{\theta_{3}^{(2)}+2 l \pi}{\omega_{12}}, \quad \tau_{l, 4}^{(2)}=\frac{\theta_{4}^{(2)}+2 l \pi}{\omega_{22}}, \quad l=0,1,2, \ldots
$$


with

$$
\theta_{3}^{(2)}=2 \pi-\cos ^{-1}\left(\frac{A-2 d}{2 d}\right) \in\left(\frac{3 \pi}{2}, 2 \pi\right) \quad \text { and } \quad \theta_{4}^{(2)}=\cos ^{-1}\left(\frac{A-2 d}{2 d}\right) \in\left(0, \frac{\pi}{2}\right) .
$$

For $\left\{\tau_{0, j}^{(k)}\right\},(k=0,2, j=1,2,3,4)$, we have the following relation.

Lemma 4.1 For $\left\{\tau_{0, j}^{(k)}\right\}(k=0,2, j=1,2,3,4)$ obtained above, $\tau_{0,4}^{(2)}<\tau_{0,1}^{(0)}<\tau_{0,2}^{(0)}<\tau_{0,3}^{(2)}$.

Proof It follows from the definitions of $\left\{\tau_{0, j}^{(k)}\right\}$ that $\tau_{0,2}^{(0)}<\tau_{0,3}^{(2)}, \tau_{0,4}^{(2)}<\tau_{0,1}^{(0)}$ and $\tau_{0,4}^{(2)}<\tau_{0,3}^{(2)}$. Note that the characteristic equation (4.2) has two roots with positive real parts when $\tau=0$. This, together with $\left.\frac{d \operatorname{Re}(\lambda)}{d \tau}\right|_{\lambda=i \omega_{1}}<0$ and $\left.\frac{d \operatorname{Re}(\lambda)}{d \tau}\right|_{\lambda=i \omega_{2}}>0$, implies that $\tau$ increases. In order for the characteristic equation to have the first pair of purely imaginary roots $\pm i \omega$, there must be $\left.\frac{d \operatorname{Re}(\lambda)}{d \tau}\right|_{\lambda=i \omega}<0$. This implies $\omega=\omega_{1}$ and hence $\tau_{0,1}^{(0)}<\tau_{0,2}^{(0)}$.

The case $d>\frac{A}{2}$ can be handled similarly to the case with $\frac{A}{4}<d<\frac{A}{2}$. Thus, we can obtain four sequences $\left\{\tau_{l, 5}^{(0)}\right\},\left\{\tau_{l, 6}^{(0)}\right\},\left\{\tau_{l, 7}^{(2)}\right\},\left\{\tau_{l, 8}^{(2)}\right\}$ defined by

$$
\begin{array}{ll}
\tau_{l, 5}^{(0)}=\frac{\theta_{5}^{(0)}+2 j \pi}{\omega_{1}}, & \theta_{5}^{(0)}=\cos ^{-1}\left(\frac{2 d-A}{2 d}\right) \in(0, \pi / 2), \\
\tau_{l, 6}^{(0)}=\frac{\theta_{6}^{(0)}+2 j \pi}{\omega_{2}}, & \theta_{6}^{(0)}=2 \pi-\theta_{5}^{(0)} \in(3 \pi / 2,2 \pi), \\
\tau_{l, 7}^{(2)}=\frac{\theta_{7}^{(2)}+2 j \pi}{\omega_{1}}, & \theta_{7}^{(2)}=2 \pi-\cos ^{-1}\left(\frac{2 d-A}{2 d}\right) \in(\pi, 3 \pi / 2), \\
\tau_{l, 8}^{(2)}=\frac{\theta_{8}^{(2)}+2 j \pi}{\omega_{2}}, & \theta_{8}^{(2)}=\cos ^{-1}\left(\frac{2 d-A}{2 d}\right) \in(\pi / 2, \pi),
\end{array}
$$

where $l=0,1,2, \ldots$.

By the definitions of $\left\{\tau_{0, j}^{(k)}\right\}$, we have the following relations.

Lemma 4.2 For $\left\{\tau_{0, j}^{(k)}\right\}(k=0,2, j=5,6,7,8)$ obtained above, we have $\tau_{0,8}^{(2)}<\tau_{0,7}^{(2)}, \tau_{0,5}^{(0)}<\tau_{0,6}^{(0)}$, $\tau_{0,5}^{(0)}<\tau_{0,7}^{(2)}$ and $\tau_{0,8}^{(2)}<\tau_{0,6}^{(0)}$. Further, if $\tau_{0,8}^{(2)}<\tau_{0,5}^{(0)}$, then $\tau_{0,8}^{(2)}<\tau_{0,5}^{(0)}<\tau_{0,6}^{(0)}<\tau_{0,7}^{(2)}$.

Proof Similar to the proof of Lemma 4.1, it is easy to obtain $\tau_{0,8}^{(2)}<\tau_{0,7}^{(2)}, \tau_{0,5}^{(0)}<\tau_{0,6}^{(0)}, \tau_{0,5}^{(0)}<\tau_{0,7}^{(2)}$ and $\tau_{0,8}^{(2)}<\tau_{0,6}^{(0)}$.

Next we show that if $\tau_{0,8}^{(2)}<\tau_{0,5}^{(0)}$, then $\tau_{0,6}^{(0)}<\tau_{0,7}^{(2)}$. If not, $\tau_{0,6}^{(0)}>\tau_{0,7}^{(2)}$. By the definitions of $\left\{\tau_{0, j}^{(k)}\right\}(j=5,6,7,8)$, we have $\frac{2 \pi-\theta_{5}^{(0)}}{\pi+\theta_{5}^{(0)}}>\frac{\omega_{2}}{\omega_{1}}$. Since $\tau_{0,8}^{(2)}<\tau_{0,5}^{(0)}, \frac{\pi-\theta_{5}^{(0)}}{\theta_{5}^{(0)}}<\frac{\omega_{2}}{\omega_{1}}$. Therefore, $\frac{\pi-\theta_{5}^{(0)}}{\theta_{5}^{(0)}}<$ $\frac{2 \pi-\theta_{5}^{(0)}}{\pi+\theta_{5}^{(0)}}$, which leads to $\theta_{5}^{(0)}>\pi / 2$. This is a contradiction. Thus, $\tau_{0,8}^{(2)}<\tau_{0,5}^{(0)}<\tau_{0,6}^{(0)}<\tau_{0,7}^{(2)}$ provided that $\tau_{0,8}^{(2)}<\tau_{0,5}^{(0)}$.

It follows from the results obtained in Sect. 3 that

$$
S_{2}=\left\{s_{j} \mid s_{j}=-2, j \in\{1,2, \ldots\}\right\}
$$

and

$$
S_{3}=\left\{s_{j} \mid s_{j}=-1, j \in\{1,2, \ldots\}\right\} .
$$


Theorem 4.3 Consider system (2.2) with $n=4$ and $\alpha=0$. If $A<0$, the coexistence equilibrium $E_{n}^{*}$ is locally asymptotically stable for all $\tau \geq 0$. If $A>0$, then the coexistence equilibrium $E_{n}^{*}$ is unstable when $\tau=0$, and as $\tau$ increases, we have the following results.

Case (i) $d \in(0, A / 4)$ : the coexistence equilibrium $E_{n}^{*}$ remains unstable for $\tau>0$;

Case (ii) $d \in(A / 4, A / 2)$ : the coexistence equilibrium $E_{n}^{*}$ remains unstable for $\tau>0$;

Case (iii) $d \in(A / 2, \infty)$ : the coexistence equilibrium $E_{n}^{*}$ remains unstable for $\tau>0$ provided that $\tau_{0,8}^{(2)}<\tau_{0,5}^{(0)}$ and there are $\mathcal{N} \geq 1$ stability intervals at which the coexistence equilibrium $E_{n}^{*}$ is locally asymptotically stable provided that $\tau_{0,5}^{(0)}<\tau_{0,8}^{(2)}$.

Proof Based on the above analysis, we only need to consider cases (ii)-(iii). For case (ii), by Lemma 4.1, the characteristic equations (4.2)-(4.4) admit purely imaginary roots at $\tau=\tau_{j}$, where

$$
\left\{\tau_{j}\right\}_{j=1}^{\infty}=\left\{\tau_{0,4}^{(2)}, \tau_{0,1}^{(0)}, \tau_{0,2}^{(0)}, \tau_{0,3}^{(2)}, \tau_{1,4}^{(2)}, \ldots\right\}
$$

The sequence $\left\{\tau_{j}\right\}_{j=1}^{\infty}$ has an associated sequence

$$
\left\{\sigma_{j}\right\}_{j=1}^{\infty}=\{+1,-1,+1,-1,+1, \ldots\}
$$

Then

$$
s_{1}=1, \quad s_{2}=0, \quad s_{3}=1, \quad s_{4}=0, \quad s_{5}=1, \quad \ldots .
$$

Note that, for the sequence $\left\{\tau_{l, j}^{(k)}\right\}_{k=0}^{\infty}$, the spacing between delays is $\tau_{l+1, j}^{(k)}-\tau_{l, j}^{(k)}=\frac{2 \pi}{\omega_{i k}}$, for $j=1,2,3,4$ and $i=1,2$. By the expression of $\omega_{i k}$, we have

$$
\frac{2 \pi}{\omega_{1}}>\frac{2 \pi}{\omega_{2}}
$$

This indicates in the sequence $\left\{\sigma_{j}\right\},+1$ 's appear more frequently than -1 's do. Hence $s_{j} \geq 0$ for $j=1,2, \ldots$. Consequently, the coexistence equilibrium $E_{n}^{*}$ remains unstable for $\tau>0$.

For case (iii), by Lemma 4.2, we know that for the obtained four sequences of critical values $\left\{\tau_{k, j}^{(k)}\right\}_{k=0}^{\infty}, j=5,6,7,8$, we either have (iii.a) $\tau_{0,8}^{(2)}<\tau_{0,5}^{(0)}$ or (iii.b) $\tau_{0,5}^{(0)}<\tau_{0,8}^{(2)}$. If $\tau_{0,8}^{(2)}<\tau_{0,5}^{(0)}$, then we have $\left\{\tau_{j}\right\}_{j=1}^{\infty}=\left\{\tau_{0,8}^{(2)}, \tau_{0,5}^{(0)}, \tau_{0,6}^{(0)}, \tau_{0,7}^{(2)}, \tau_{1,8}^{(2)}, \ldots\right\}$ and $\left\{\sigma_{j}\right\}_{j=1}^{\infty}=\{+1,-1,+1,-1,+1, \ldots\}$. By virtue of (4.5), we know that the coexistence equilibrium $E_{n}^{*}$ remains unstable for $\tau>0$.

If $\tau_{0,5}^{(0)}<\tau_{0,8}^{(2)}$, then the newly sorted sequence $\left\{\tau_{j}\right\}$ begins with $\tau_{1}=\tau_{0,5}^{(0)}$ and the associated sequence $\left\{\sigma_{j}\right\}_{j=1}^{\infty}$ starts with $\sigma_{1}=-1$. Hence $s_{1}=-1$ and $\mathcal{N} \geq 1$. It follows from the relation (4.5) that delays with a positive jump appear more frequently than delays with a negative jump in the sequence $\left\{\tau_{j}\right\}$ do. Thus, regardless of the ordering in the rest of the sequence $\left\{\tau_{j}\right\}$, there must exist a finite positive number $j_{0}$ such that $s_{j} \geq 0$ for $j>j_{0}$. Therefore, there are $\mathcal{N} \geq 1$ stability intervals at which the coexistence equilibrium $E_{n}^{*}$ is locally asymptotically stable.

The results of Theorem 4.3 can be summarized in Table 1 . 
Table 1 Local stability result of the coexistence equilibrium of ring-structured system (2.2) with $\alpha=0$ and $n=4$

\begin{tabular}{lll}
\hline & $A<0$ & $A>0$ \\
\hline $0<d<\frac{A}{4}$ & Stable & Unstable \\
$\frac{A}{4}<d<\frac{A}{2}$ & Stable & Unstable \\
$d>\frac{A}{2}$ & Stable & Unstable if $\tau_{0,8}^{(2)}<\tau_{0,5}^{(0)} ;$ \\
& & $\mathcal{N} \geq 1$ stability intervals exist if $\tau_{0,5}^{(0)}<\tau_{0,8}^{(2)}$ \\
\hline
\end{tabular}

\section{Numerical simulations}

In this section, we present some numerical simulations to demonstrate our theoretical results. We consider the following ring-structured patchy model:

$$
\left\{\begin{aligned}
\frac{d x_{i}(t)}{d t}= & \varepsilon x_{i}(t)\left(1-\frac{x_{i}(t)}{\kappa}-\frac{y_{i}(t)}{1+x_{i}(t)}\right)-2 d\left(\frac{\alpha \gamma y_{i}(t)}{1+x_{i}(t)}+(1-\alpha)\right) x_{i}(t) \\
& +d\left(\frac{\alpha \gamma y_{i+1}(t-\tau)}{1+x_{i+1}(t-\tau)}+(1-\alpha)\right) x_{i+1}(t-\tau)+d\left(\frac{\alpha \gamma y_{i-1}(t-\tau)}{1+x_{i-1}(t-\tau)}+(1-\alpha)\right) x_{i-1}(t-\tau), \\
\frac{d y_{i}(t)}{d t}= & \frac{x_{i}(t) y_{i}(t)}{1+x_{i}(t)}-\mu y_{i}(t)
\end{aligned}\right.
$$

for $i=1,2,3,4$. To close the ring, define $x_{0}=x_{4}, x_{5}=x_{1}, y_{0}=y_{4}$ and $y_{5}=y_{1}$.

We take parameter values $\varepsilon=1, \kappa=2, \mu=0.2, n=4, \gamma=0.5, d=0.04, \alpha=0$. This set of parameter values corresponds to the case $\alpha=0$ and $d>A / 2=0.05 / 2=0.025$, and the obtained four sequences of critical values of $\tau$ are

$$
\begin{array}{ll}
\tau_{l, 5}^{(0)} \approx 3.50+18.54, & \tau_{l, 6}^{(0)} \approx 12.34+15.21, \\
\tau_{l, 7}^{(2)} \approx 12.77+18.54, & \tau_{l, 8}^{(2)} \approx 4.73+15.21,
\end{array}
$$

for $l=0,1, \ldots$ Thus the sorted sequence of critical values of $\tau$ reads

$$
\left\{\tau_{j}\right\}_{j=1}^{\infty}=\{3.50,4.73,12.34,12.77,19.94, \ldots\}
$$

Therefore,

$$
\left\{s_{j}\right\}_{j=1}^{\infty}=\{-1,0,1,0,1, \ldots\}
$$

and we have an $\mathcal{N}=1$ stability interval. The coexistence equilibrium $E_{n}^{*}$ is locally asymptotically stable for $\tau \in\left(\tau_{1}, \tau_{2}\right)=(3.50,4.73)$. A bifurcation diagram is presented in Fig. 1 .

Next we fix $\alpha=0.5$ and vary the value of the dispersal rate $d$. We find that the number of stability intervals changes from 0 to 1 near $d=0.0421$, then it changes to 2 when $d=$ 0.0721 , and back to 1 at about $d=0.2651$. This shows that the dispersal rate can also induce finite number of stability switches. This is illustrated in Fig. 2 .

Now we fix $d=0.13$ and vary the value of $\alpha$. We find that the number of stability intervals changes from 1 to 2 and back to 1 near $\alpha=0.023$ and $\alpha=0.939$, respectively (see Fig. 3). This indicates that the fraction of prey dispersing due to predation avoidance can also induce stability switches.

For the case with $\alpha>0$ and $A<0$, we take parameter values $\kappa=1.8, \mu=0.3, \varepsilon=1.1$, $n=4, \alpha=0.6, d=0.6, \gamma=0.5$. Regarding $\tau$ as the bifurcation parameter, we find that there are $\mathcal{N}=30$ stability intervals. We show some of them in Fig. 4 . 


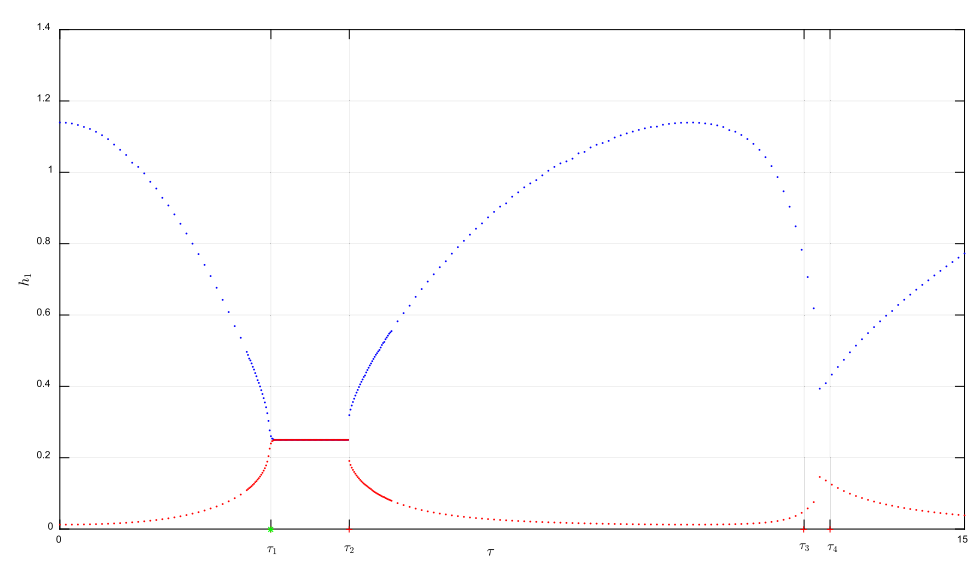

Figure 1 Bifurcation diagram of ring-structured patchy model (5.1) with $\alpha=0$ and $d>A / 2$. There is $\mathcal{N}=1$ stability interval. Parameter values used are $\kappa=2, \mu=0.2, \varepsilon=1, n=4, \gamma=0.5, \alpha=0, d=0.04$
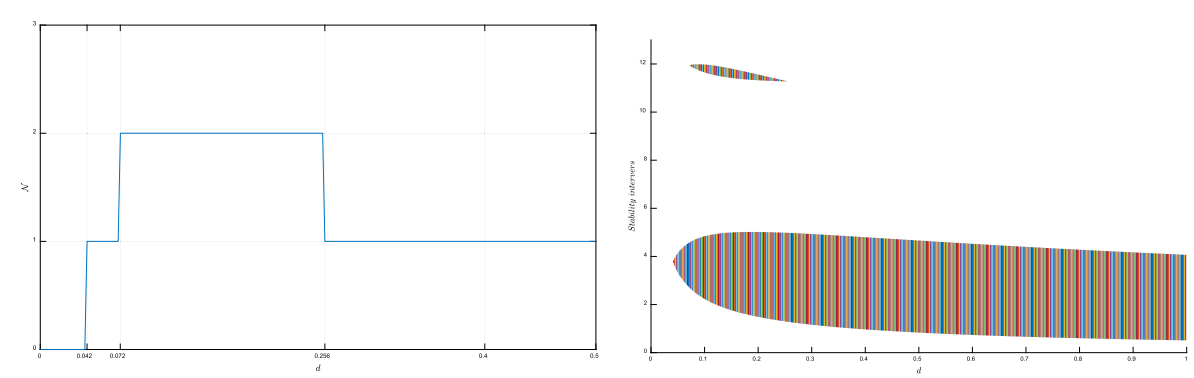

Figure 2 The effect of dispersal rate $d$. The left panel: the number of stability intervals of system (5.1); the right panel: the distribution of stability intervals system (5.1). The parameter values are $\alpha=0.5, \epsilon=1, \kappa=2$, $\mu=0.2, \gamma=0.5, n=4$
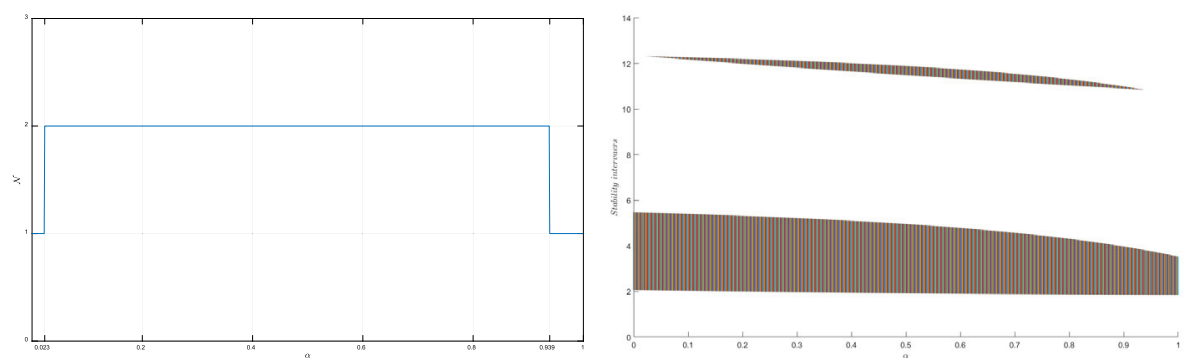

Figure 3 The effect of $\alpha$ when $d=0.13$ is fixed. The left panel: the number of stability intervals of system (5.1); the right panel: the distribution of stability intervals of system (5.1). Other parameter values are the same as in Fig. 2

Next, using Figs. 5 and 6, we illustrate that the configuration of network topology also has an impact on the stability switches of the coexistence equilibrium by comparing our ringstructured patchy model with the fully connected model studied in [18]. The differences are significant when $n$ is odd, and when $\alpha$ is small and $d$ is large. 


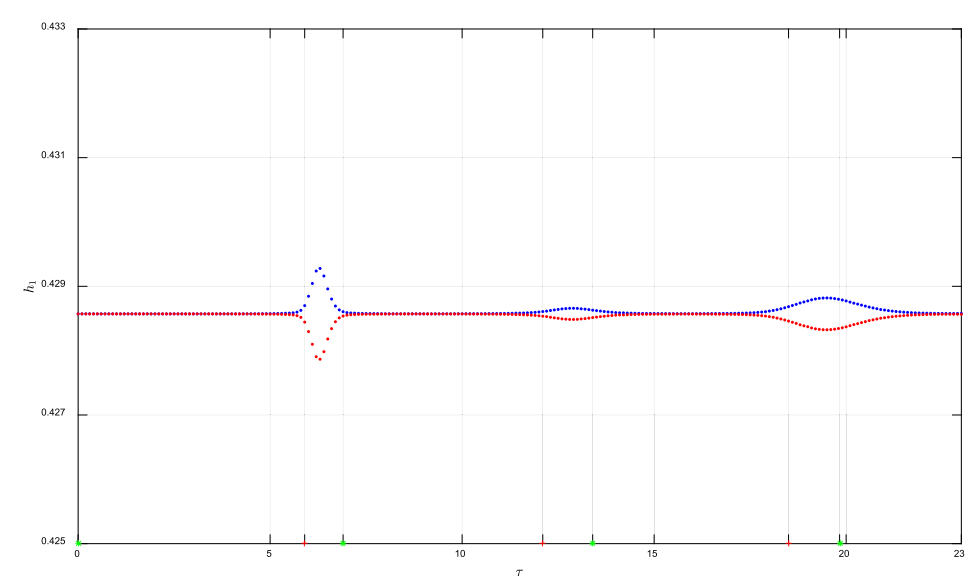

Figure 4 Bifurcation diagram of ring-structured patchy model (5.1) under case $\alpha>0$ and $A<0$. The parameter values are $\kappa=1.8, \mu=0.3, \varepsilon=1.1, n=4, \alpha=0.6, d=0.6, \gamma=0.5$



\section{Summary}

In this paper, we have considered a metapopulation predator-prey model with delayed dispersal within a ring-structured configuration of network topology. Prey in one patch are assumed to move between two neighboring patches on the ring. We have shown that the dispersal delay can induce stability switches for the case with random dispersal only and also for the case with random dispersal and density-dependent dispersal. We have also shown that the dispersal rate $(d)$ and the fraction of dispersal due to predation avoidance $(\alpha)$ can induce stability switches. In addition, by comparing our ring-structured patchy model with the fully connected model studied in [18], we find that the configuration of network topology also has an impact on the stability switches of the coexistence equilib- 


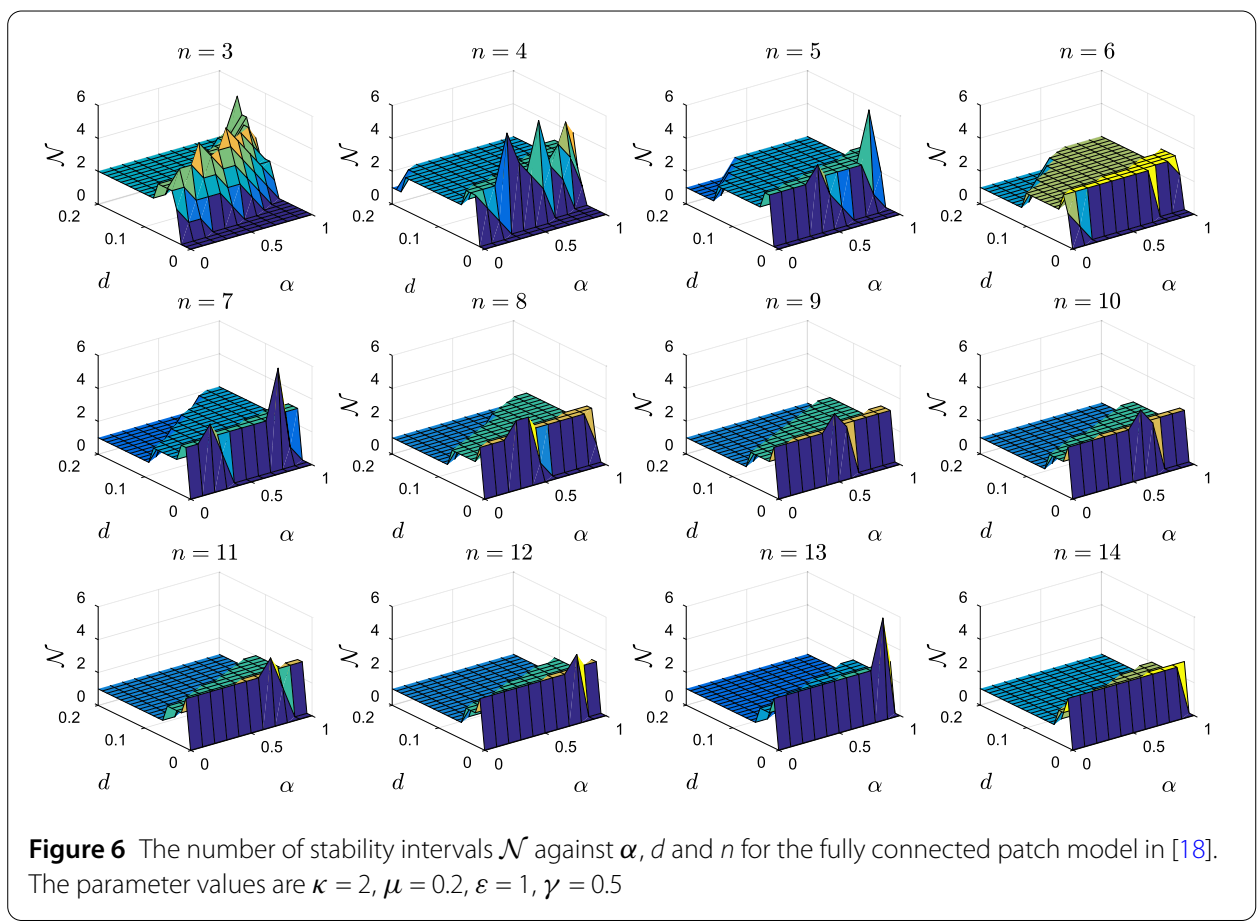

rium, especially when the number of patches $n$ is odd, the fraction $\alpha$ of dispersal due to predation avoidance is small and the dispersal rate $d$ is large.

\section{Acknowledgements}

The authors would like to thank the editor and the referees for their valuable suggestions, which helped to improve this work.

\section{Funding}

This work was supported by Scientific and Technological Innovation Programs of Higher Education Institutions in Shanxi (No.2019L0866, No.2019L0848), by the Natural Science Foundation of Shanxi (No. 201901D111295), and by the Research Foundation of Yuncheng University (No. YQ-2017003, No.XK-2018033).

\section{Availability of data and materials}

Please contact the corresponding author for data requests.

\section{Competing interests}

The authors declare that they have no competing interests.

\section{Authors' contributions}

All authors contributed equally to the writing of this paper. The authors read and approved the final manuscript.

\section{Publisher's Note}

Springer Nature remains neutral with regard to jurisdictional claims in published maps and institutional affiliations.

Received: 15 January 2020 Accepted: 13 April 2020 Published online: 05 May 2020

\section{References}

1. Murray, J.D.: Mathematical Biology. Springer, New York (2002)

2. Hanski, I.: Metapopulation dynamics. Nature 396(6706), 41 (1998)

3. Shigesada, N., Kawasaki, K.: Biological Invasions: Theory and Practice. Oxford University Press, Oxford (1997)

4. Pillai, P., Gonzalez, A., Loreau, M.: Evolution of dispersal in a predator-prey metacommunity. Am. Nat. 179(2), 204-216 (2011)

5. Freedman, $H_{\text {.: }}$ Single species migration in two habitats: persistence and extinction. Math. Model. 8, $778-780$ (1987)

6. Freedman, H., Takeuchi, Y.: Global stability and predator dynamics in a model of prey dispersal in a patchy environment. Nonlinear Anal., Theory Methods Appl. 13(8), 993-1002 (1989)

7. Kuang, Y., Takeuchi, Y.: Predator-prey dynamics in models of prey dispersal in two-patch environments. Math. Biosci. 120(1), 77-98 (1994) 
8. Wang, X., Zou, X:: On a two-patch predator-prey model with adaptive habitancy of predators. Discrete Contin. Dyn. Syst., Ser. B 21(2), 677-697 (2016)

9. Kang, Y., Sourav, K., Komi, M.: A two-patch prey-predator model with predator dispersal driven by the predation strength. Math. Biosci. Eng. 14(4), 843-880 (2017)

10. Abdllaoui, A., Auger, P., Kooi, B., Parra, R., Mchich, R.: Effects of density-dependent migrations on stability of a two-patch predator-prey model. Math. Biosci. 210(1), 335-354 (2007)

11. Mchich, R., Auger, P., Poggiale, J.: Effect of predator density dependent dispersal of prey on stability of a predator-prey system. Math. Biosci. 206(2), 343-356 (2007)

12. Wang, W., Takeuchi, Y:: Adaptation of prey and predators between patches. J. Theor. Biol. 258(4), 603-613 (2009)

13. Feng, W., Rock, B., Hinson, J.: On a new model of two-patch predator prey system with migration of both species. J. Appl. Anal. Comput. 1(2), 193-203 (2011)

14. Sun, G., Mai, A.: Stability analysis of a two-patch predator-prey model with two dispersal delays. Adv. Differ. Equ. 2018(1), Article ID 373 (2018)

15. Sun, G., Mai, A.: Stability analysis of a two-patch competition model with dispersal delays. Discrete Dyn. Nat. Soc. 2019, 1-10 (2019)

16. Neubert, M., Klepac, P., Driessche, P.: Stabilizing dispersal delays in predator-prey metapopulation models. Theor. Popul. Biol. 61(3), 339-347 (2002)

17. Zhang, Y., Lutscher, F., Guichard, F.: The effect of predator avoidance and travel time delay on the stability of predator-prey metacommunities. Theor. Ecol. 8(3), 273-283 (2015)

18. Mai, A., Sun, G., Zhang, F., Wang, L.: The joint impacts of dispersal delay and dispersal patterns on the stability of predator-prey metacommunities. J. Theor. Biol. 462(2), 455-465 (2019)

19. Mai, A., Sun, G., Wang, L.: Impacts of the dispersal delay on the stability of the coexistence equilibrium of a two-patch predator-prey model with random predator pispersal. Bull. Math. Biol. 81(5), 1337-1351 (2019)

20. Grant, E., Lowe, W., Fagan, W.: Living in the branches: population dynamics and ecological processes in dendritic networks. Ecol. Lett. 10(2), 165-175 (2007)

21. Hsu, S.: On global stability of a predator-prey system. Math. Biosci. 39(1-2), 1-10 (1978)

22. Labine, P.A., Wilson, D.H.: A teaching model of population interactions: an algae-Daphnia-predator system. Bioscience 23(3), 162-167 (1973)

23. Friedman, B.: Eigenvalues of composite matrices. Math. Proc. Camb. Philos. Soc. 57(1), 37-49 (1961)

24. Edelstein-Keshet, L:: Mathematical Models in Biology. Classics in Applied Mathematics, vol. 46. SIAM, Philadelphia (1988)

25. Hale, J., Lunel, S.: Introduction to Functional Differential Equations. Applied Mathematical Sciences, vol. 99. Springer, Berlin (2013)

26. Cooke, K., Grossman, Z:: Discrete delay, distributed delay and stability switches. J. Math. Anal. Appl. 86(2), 592-627 (1982)

27. Kuang, Y.: Delay Differential Equations: With Applications in Population Dynamics. Mathematics in Science and Engineering, vol. 191. Academic Press, New York (1993)

\section{Submit your manuscript to a SpringerOpen ${ }^{\circ}$ journal and benefit from:}

- Convenient online submission

Rigorous peer review

- Open access: articles freely available online

- High visibility within the field

- Retaining the copyright to your article

Submit your next manuscript at $\gg$ springeropen.com 